\title{
The influence of R\&D intensity of countries on the impact of international collaborative research: Evidence from Spain
}

\author{
María Bordons ${ }^{1}$, Borja González-Albo², Javier Aparicio², Luz Moreno \\ ${ }^{1}$ IFS, Centre for Human and Social Sciences (CCHS), Spanish National Research \\ Council (CSIC), Albasanz 26-28, 28037 Madrid (Spain) \\ ${ }^{2}$ Centre for Human and Social Sciences (CCHS), Spanish National Research Council \\ (CSIC), Albasanz 26-28, 28037 Madrid (Spain)
}

Maria.bordons@cchs.csic.es; borja.gonzalezalbo@cchs.csic.es;

javier.aparicio@cchs.csic.es; luz.moreno@cchs.csic.es

\section{Corresponding author:}

María Bordons

Tel.34-91-602-28-76

Fax: 34-91-602-29-71

\begin{abstract}
A positive influence of international collaboration on the impact of research has been extensively described. This paper delves further into this issue and studies to what extent the type of collaborating country -high, medium or low R\&D intensive countryand which country is the leader in the research may influence the impact of the final scientific output. Among 9,961 papers co-authored by scientists from Spain and from another country (bilateral collaboration) during 2008-2009, papers with high R\&D intensive countries predominated $(60 \%)$ and received the highest number of citations. This holds true in eight out of nine fields, being Social Sciences the one which benefited the most from partnerships with high R\&D intensive countries. Mathematics emerges as a special case where other factors such as the partner's specialisation in the field may have a greater influence on research impact than the level of investment in R\&D of the collaborating country. No significant influence of the type of country leading the research on the impact of the final papers is observed in most fields. Research policy implications are finally discussed.
\end{abstract}

Keywords: international collaboration; bilateral collaboration; research impact; coauthorship analysis; Spain. 


\section{Introduction}

The positive influence of international collaboration on the impact of research has been extensively described from a bibliometric perspective (see for example, Glänzel, 2001; Katz and Hicks, 1997; Van Raan, 1997; Narin, Stevens and Whitlow, 1991) and different underlying reasons have been put forward. On the one hand, the higher number of authors and institutions described for this type of collaboration can be a key issue, since it may include a greater variety of points of view which may lead to more creativity and innovation (Reagans and Zuckerman, 2001). Moreover, innovation is expected to be strengthened by the recombination of resources from centres located in different national systems and traditions since wider diversity may lead to more original results (Nomaler, Frenken and Heimeriks, 2013). On the other hand, as the number of authors and institutions grows, so does the dissemination of the research within the personal networks of the authors thus making higher citation rates more likely (Katz and Martin, 1997; Goldfinch, Dale and DeRouen, 2003). However, differences by country and field may exist and all countries may not equally benefit from international collaboration.

According to a study on world publications covered by the Science Citation Index during 1995-1996, international co-authorship resulted in publications with higher citation rates than purely domestic papers and the influence of international collaboration on national citation impact figures varied considerably by country and by field (Glänzel, 2001). International collaboration can be particularly advantageous for less advanced countries, because they obtain access to facilities, funding, equipment and networks. Nonetheless, working with foreign partners is also beneficial for highly industrialised countries (Glänzel et al.,1999; Gorraiz, Reimann and Gumpenberger, 2012). The fact that most countries may gain from international collaboration in terms of impact was also pointed out by Adams, Gurney and Marshall (2007), in a study focused on the scientific output of the UK and its main partners. It was observed that international links were in general positive for the UK and that all the countries studied in the survey gained from collaborating with the UK in terms of impact, although differences by disciplines do exist with the highest gain reported in biomedical sciences, where the UK is a strong partner. According to Glanzel and Schubert (2004), the positive effects of international collaboration can be especially significant in fields where "targeting" is more important than "global visibility", that is, authors publish their results in their mother tongue and in domestic journals to reach a local audience (for example in clinical medicine). This local orientation may change when authors from abroad are involved and an important increase in visibility and impact may result from said involvement. More recently, a study of the scientific collaboration network including the 37 countries with the highest production in Scopus during 2003-2009 has shown that most collaborations result in gains for both sides, although countries tend to obtain greater advantage from collaborating with high-impact countries. Interestingly, the highest increase in impact in that study was obtained in Humanities, Medicine and Social Sciences, where the rate of international collaboration was very low (GuerreroBote, Olmeda-Gómez and Moya-Anegón, 2013).

The extent of the impact differences derived in internationally co-authored papers from which country is the leader in the research is also a matter of concern. Assuming that first-authorship is an indication of scientific leadership (Van Leeuwen, 2009; JiménezContreras et al., 2010), a high share of first authorships of a country in international coauthored publications could suggest it frequently plays a primary role in the research. Moreover, which is the leading country in the research may have a significant influence on the final impact of any given paper. All eight countries studied by van Leeuwen 
(2009) gained in terms of impact from international collaboration, but only in the case of the United States the impact of its first-authored papers exceeded the impact of all its internationally co-authored papers, thus suggesting a very positive influence of US leadership on paper impact. As regards Spain, lower impact was described for its firstauthored papers compared to all its internationally co-authored papers (van Leeuwen, 2009), but we do not know to what extent these results may vary by field or type of collaborating country (high or low R\&D intensive country).

In this context, this paper tries to analyse the combined influence of some of the abovementioned factors, that is, the type of collaborating country according to its level of R\&D investment and which partner takes the lead in the research, on the impact of the final scientific output. To the best of our knowledge, an in-depth analysis of the combined influence of these factors is missing in prior literature. Our underlying assumption is that teams can benefit especially from the knowledge, infrastructures and prestige of scientifically advanced countries as project partners or leaders, although differences by field may exist.

\section{Research questions}

This paper analyses the internationally co-authored papers of Spain to explore to what extent the level of investment in research and development of the collaborating country - high, medium or low level - and which is the leading country in the research may influence the impact of the resulting scientific papers.

The following questions are addressed:

- In what proportion does Spain collaborate with high and low R\&D investing countries? Are there any differences by field?

- Is there any relationship between the level of R\&D investment of the collaborating country and the impact of research? Specifically, are papers coauthored with high R\&D investing countries published in more prestigious journals? Do they receive more citations?

- Are papers lead by high R\&D investing countries published in more prestigious journals? Do they receive more citations?

These questions are studied in Spanish scientific publications including international bilateral collaboration over a two-year period and differences by field are explored.

\section{Methods}

Spanish scientific publications in 2008-2009 as covered by the Web of Science database (Science Citation Index Expanded, Social Sciences Citation Index and Arts \& Humanities Citation Index) are analysed. Only articles, proceedings papers and reviews are considered (hereinafter called "articles" or "papers" interchangeably). Papers are assigned to disciplines according to the Web of Science classification of journals into subfields. Disciplines are grouped into ten broad fields as described elsewhere (Gómez et al., 2012).

Articles with at least one foreign centre are considered internationally co-authored. The study focuses on papers involving bilateral collaboration, that is, papers developed in collaboration between Spain and just another country. Papers with multilateral collaboration (more than two countries) are not considered to have a clearer picture about the influence of the type of collaborating country by level of R\&D investment on the impact of research. Moreover, only publications with two addresses, one from 
Spain and the other from a foreign partner are taken into account to avoid the influence of the participation of additional institutions.

Countries which collaborate with Spain are classified in three classes (hereinafter called "type of country") according to their "R\&D intensity" (Eurostat, 2014), that is, their gross domestic expenditures in research and development as a percentage of their gross domestic product (\%GERD/GDP) (source of data: World Bank, 2012). R\&D intensity is a key indicator to monitor the R\&D investment level of countries and to make international comparisons. Spain devoted $1.35 \%$ of its GDP to R\&D in 2008. Accordingly, countries are classified in the following three types: a) high R\&D countries: those which are more $R \& D$ intensive than Spain according to their R\&D investment level (\%GERD/GDP above 1.55); b) low R\&D countries (\%GERD/GDP below 1.15) and c) similar to Spain $(1.15>\% \text { GERD/GDP }<1.55)^{1}$.

The impact of research is measured through two different indicators: a) Normalised journal position (NJP), which takes into account the position of the publication journal in the ranking of journals in descending order of impact factor within each subfield (Bordons and Barrigón, 1992). This indicator ranges between 0 and 1, assigning the highest values to the best positioned journals. b) Citations per paper, which consider citations received by publications with a four-year citation window. To make interfield comparisons possible, a relative citation rate $(\mathrm{RCR})$ is calculated as the citation count received by a given paper normalised with respect to the average citation rate of Spain in the discipline of the publication journal. Values above 1 are obtained by papers which have received more citations than the country's average in the discipline, while the opposite holds for values below 1.

Several factors such as the number of references or the number of authors which sign a paper may have an influence on the final impact of the research. A high number of references may result from very comprehensive research papers (McVeigh \& Mann, 2009; González-Albo \& Bordons, 2011; Costas et al., 2012) and may contribute to the quality of publications as well as, in the long term, attract a higher number of citations (Haslam et al., 2008). Concerning the number of authors, research can benefit from the collaboration between authors who may contribute with their different specialisation profiles and skills to the development of the research. In fact, a positive correlation between number of authors and research impact has been formerly described in the literature (Gazni and Didegah, 2011).

In order to study the role of Spain as leader of the research the usefulness of two different approaches were initially assessed. "First authorship" could be considered as an indication of scientific leadership (Van Leeuwen, 2009) and accordingly, the country of the first author would be the deciding factor. However, since the criteria for determining the order of the authors in the byline may change by field (Frandsen and Nicolaisen, 2010; Marusic, Bosnjak and Jeroncic, 2011; Wren et al., 2007) and the alphabetical order of authors is the norm in a few disciplines such as Mathematics and Economics (Waltman, 2012), we decided to assign leadership to the country of the corresponding author. Initially, the corresponding author simply indicated the group

\footnotetext{
1 a)High R\&D countries: Australia, Austria, Belgium, Canada, Denmark, Finland, France, Germany, Israel, Japan, Luxembourg, Netherlands, Norway, Singapore, Slovenia, South Korea, Sweden, Switzerland, United Kingdom, United States of America. b) Low R\&D countries: Ethiopia, Greece, Guatemala, Hungary, India, Iran, Jordan, Kuwait, Latvia, Lithuania, Malaysia, Mexico, Moldova, Morocco, Mozambique, Pakistan, Panama, Paraguay, Philippines, Poland, Romania, Russia, Saudi Arabia, Senegal, Serbia, Slovakia, South Africa, Tanzania, Thailand, Trinidad \& Tobago, Tunisia, Turkey, Ukraine, Uruguay, Zambia. c) Similar to Spain countries: China, Czech Republic, Estonia, Ireland, Italy, New Zealand, Portugal. Note: only countries included in the Spanish publications are shown.
} 
member chosen to be responsible for all contact and correspondence purposes with the journal (Mattsson, Sundberg and Laget, 2011), but gradually the corresponding author has become widely accepted as a particularly important author with leadership and responsibility functions (Wren et al., 2007). Further evidence of the importance of the corresponding author lies with the fact that they most frequently appear first in the byline with last position as the second most frequent choice (Mattsson, Sundberg and Laget, 2011). It is interesting to remark that in our set of international bilateral coauthored papers, the country of the first address matches the country of the corresponding author in $97 \%$ of the articles, supporting our assumption on the key role played by both positions in research.

Since the relative citation rate shows a skewed distribution, it is categorized. Four different classes of papers are considered: non-cited $(\mathrm{RCR}=0)$; low to moderate citation rate (RCR higher than 0 and lower than 1 ); moderate to high citation rate (RCR equal or higher than 1 but lower than 2); and high citation rate (RCR equal or higher than 2). Ordinal logistic regression is used to analyse relationships between the relative citation rate and several independent variables. Ordinal logistic regression is a simple extension of binary logistic regression that allows for more than two categories of the dependent or outcome variable (four categories in this case) to be ordered (e.g., from low to high). Ordinal logistic regression is often considered an attractive analysis because it does not assume normality, linearity, or homogeneity of variance for the independent variables (Garson, 2013).

The association between the relative citation rate and the following variables is analysed: type of country (1=high R\&D country; $2=$ similar to Spain; $3=$ low R\&D country), country of the corresponding author (coded as $1=$ Spain, $0=$ other countries), number of authors, number of references, normalised journal position and research field (ten fields ${ }^{2}$ ). Since a given paper may be included in more than one field due to the multi-assignation of journals into more than one field, nine dummy coded variables are created (one dummy per field, Humanities is excluded) (e.g., the AGRI variable is coded as 1 for papers in Agriculture and 0 for the rest of the papers). Humanities is not considered in this analysis because of the limited value of citations in this field. Since the influence of type of country and country of the corresponding author on the impact of the research may vary by fields, the interactions between these independent variables and each of the 9 fields considered are explored. SPSS (v.20) is used for the statistical analysis of data.

\section{Results}

A total of 84,333 papers were published by Spain in $2008-2009$. Around $71 \%$ of them were conducted in collaboration between two or more centres and $41 \%$ included at least one foreign partner. Considering internationally co-authored papers, multilateral collaboration was present in 12,094 papers (35\% of internationally co-authored papers), while bilateral collaboration was the case in 22,515 papers (65\%). As previously described in the literature, the presence of collaboration varies by field: the lowest values are found in Humanities and Social Sciences and the highest in Physics, Biomedicine and Clinical Medicine. Physics is the field with the highest percentage of internationally co-authored papers (64\%), followed by the Multidisciplinary field $(53 \%)$. Bilateral collaboration predominates over multilateral collaboration in all fields, although some interfield differences are observed: multilateral collaboration comprises more

\footnotetext{
${ }^{2}$ Agriculture, Biology and Environment (AGRI); Biomedicine (BIOM); Chemistry (CHEM); Clinical Medicine (CLIN); Engineering/Technology (ENGI); Humanities (HUMA); Mathematics (MATH); Multidisciplinary (MULT); Physics (PHYS) and Social Sciences (SOCl).
} 
than $40 \%$ of the internationally co-authored papers in the Clinical Medicine, Physics and Multidisciplinary fields, while it is below $30 \%$ in most of the remaining fields, with the exception of Biomedicine which shows intermediate values. Special emphasis should be placed on the high values of bilateral collaboration in Humanities and Mathematics, where around $80 \%$ of internationally co-authored papers present a bilateral scheme (table 1).

Table 1. Scientific papers with Spanish participation by field and type of collaboration, WoS 2008-2009

\begin{tabular}{|l|c|c|c|c|c|c|}
\hline \multirow{2}{*}{ Fields } & Total & \multicolumn{3}{|c|}{ \% Articles by type of collab. } & \multicolumn{2}{c|}{ Internat. Collab. } \\
\cline { 3 - 6 } & N.Art. & International & National & No coll. & Bilateral & Multilateral \\
\hline Agric.Biol. Environment & 16,074 & 40.30 & 41.17 & 30.02 & 71.50 & 28.50 \\
Biomedicine & 16,170 & 42.44 & 52.07 & 21.76 & 64.20 & 35.80 \\
Chemistry & 11,782 & 40.51 & 38.74 & 32.42 & 75.95 & 24.05 \\
Clinical Medicine & 18,441 & 31.56 & 60.00 & 21.86 & 52.70 & 47.30 \\
Engineering/Technology & 16,834 & 40.29 & 36.13 & 34.04 & 73.83 & 26.17 \\
Humanities & 2,299 & 11.92 & 11.35 & 79.08 & 83.58 & 16.42 \\
Mathematics & 4,748 & 42.86 & 31.70 & 34.22 & 80.20 & 19.80 \\
Multidisciplinary & 1,052 & 52.76 & 36.60 & 29.66 & 56.76 & 43.24 \\
Physics & 14,436 & 63.56 & 41.18 & 18.15 & 55.99 & 44.01 \\
Social Sciences & 7,374 & 30.28 & 33.03 & 45.38 & 74.61 & 25.39 \\
\hline Total Spain & 84,333 & 41.04 & 43.16 & 29.28 & 65.06 & 34.94 \\
\hline
\end{tabular}

Note: papers including both national and international collaboration are considered in the "international" category. The "No collaboration" category includes papers with a single address.

Internationally co-authored papers were published in more prestigious journals (higher NJP) and received a higher number of citations than the other two collaborative categories of papers $\left(p<0.01^{3}\right)$ both in the total Spanish output and in each individual field. The impact relative to the country average by type of collaboration is shown in figure 1 to make interfield comparisons possible. The citation rate of the papers with foreign partners was above the Spanish average in all fields (relative citation rate $>1$ ). Clinical Medicine, Multidisciplinary, Social Sciences and Biomedicine were the fields which benefited most from international collaboration in terms of the citation increase associated to this type of collaboration (citations rates at least $30 \%$ higher than the country's average citation rate in each field). However, it should be noted that the high values of relative citation rates observed for internationally co-authored papers in Clinical Medicine and Social Sciences are bolstered by the relatively low share of papers with international collaboration in both disciplines (around $30 \%$ vs. $41 \%$ in terms of total country figures), thus accounting for the lower influence of these papers on the average citation rate for each discipline and contributing to make more evident the increase due to international links.

Internationally co-authored papers with more than two institutional addresses were published in more prestigious journals (higher NJP) and received more citations than those including only two partners $\left(<0.001^{4}\right)$. The impact relative to the country average by number of addresses and field is shown in figure 2 . This finding is consistent with previous research conducted on Austria and other six countries (Gorraiz, Reimann and Gumpenberger, 2012), where domestic publications were less cited than bilateral ones, while the latter were, in turn, less cited than multilateral ones.

\footnotetext{
${ }^{3}$ Kruskal-Wallis test for non-parametric distributions

${ }^{4}$ Mann-Whitney test for non-parametric distributions
} 
Figure 1. Impact relative to the country average by type of collaboration and field (Spain, WoS 2008-2009)
a. Citations
b. Normalised Journal Position
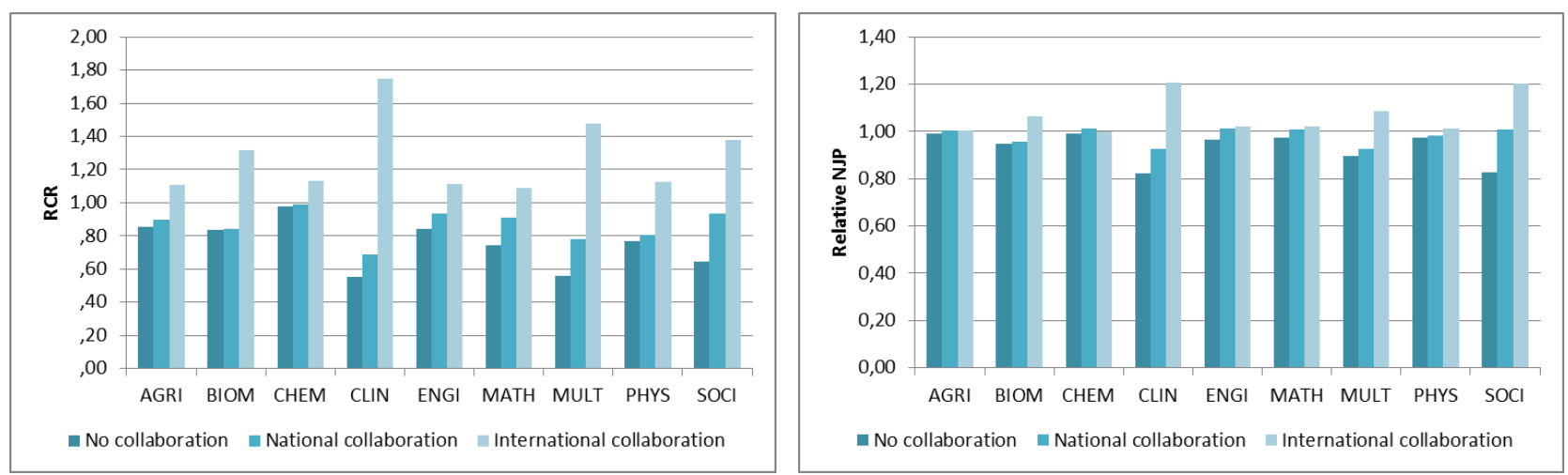

Figure 2. Impact relative to the country average of international collaboration: bilateral vs. multilateral collaboration (Spain, WoS 2008-2009)
a. Citations
b. Normalised Journal Position
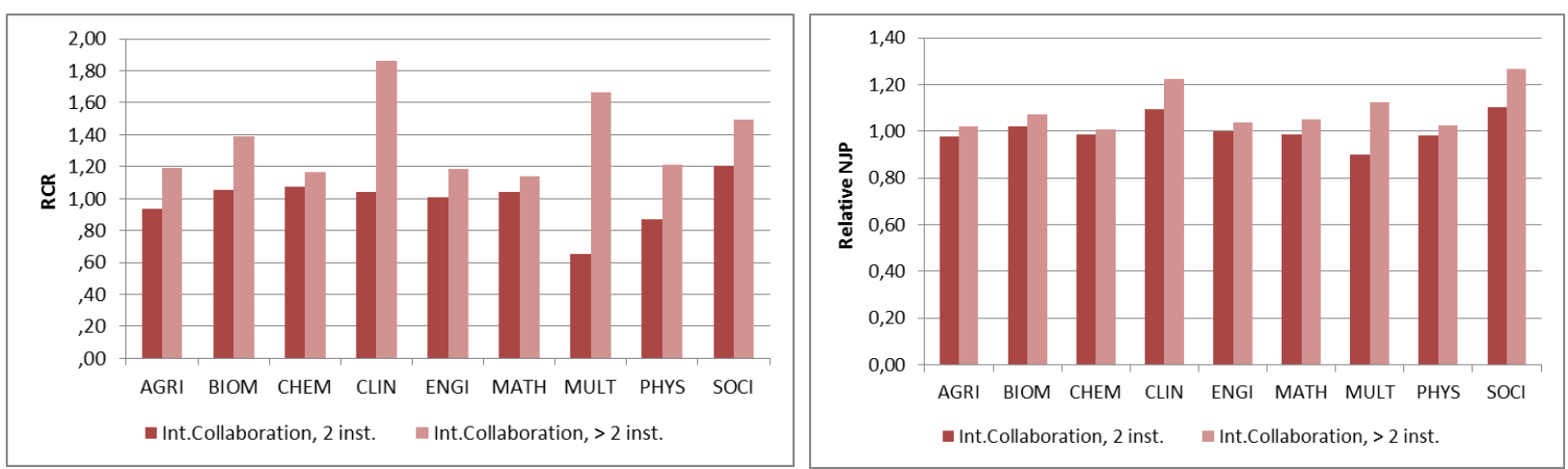


\subsection{Mean features of international bilateral publications}

A total of 9,961 publications included two centres from two different countries, one of them being Spain. On average, $60 \%$ of these papers were developed with high R\&D countries, while $25 \%$ included low R\&D countries and $14 \%$ were co-authored with authors from countries with R\&D investment figures similar to that of Spain. Table 2 shows differences by field. High R\&D countries predominate across all fields, but their presence is well above average $(>70 \%)$ in the Multidisciplinary field, Social Sciences and Humanities, while the lowest values are observed in Chemistry (50\%), Agriculture $(54 \%)$ and Mathematics (56\%). The percentage of papers in collaboration with countries whose R\&D intensity is similar to that of Spain ("medium R\&D countries" in table 2) ranges from $10 \%$ to $15 \%$ in most fields. Around $56 \%$ of the papers showed a corresponding author from Spain, although this percentage ranges from $53 \%$ in Clinical Medicine to $60 \%$ in Agriculture.

Table 2. Publications with Spanish participation in bilateral international collaboration: number of articles by field, distribution of articles by type of collaborating country and share of articles with a corresponding author from Spain (WoS, 2008-2009)

\begin{tabular}{|l|r|c|c|c|c|}
\hline \multirow{2}{*}{ Field } & \multirow{2}{*}{ N.Art. } & \multicolumn{2}{c|}{ \% Art. By type of collaborating country } & \% Art. with \\
\cline { 3 - 6 } & & High R\&D & Medium R\&D & Low R\&D & $\begin{array}{l}\text { corresponding } \\
\text { author from Spain }\end{array}$ \\
\hline Agric., Biol. , Environment & 2,102 & 54.11 & 17.02 & 28.88 & 59.68 \\
Biomedicine & 1,452 & 69.84 & 12.46 & 17.70 & 56.18 \\
Chemistry & 1,772 & 50.49 & 16.71 & 32.80 & 55.08 \\
Clinical Medicine & 780 & 63.42 & 14.40 & 22.18 & 52.72 \\
Engineering/Technology & 2,644 & 59.75 & 12.85 & 27.40 & 57.60 \\
Humanities & 146 & 70.83 & 11.11 & 18.06 & 54.93 \\
Mathematics & 1,024 & 55.57 & 17.05 & 27.38 & 55.25 \\
Multidisciplinary & 102 & 73.74 & 4.04 & 22.22 & 52.94 \\
Physics & 2,192 & 62.85 & 13.72 & 23.43 & 53.86 \\
Social Sciences & 905 & 72.85 & 10.18 & 16.97 & 57.33 \\
\hline Total & 9,961 & 60.45 & 14.25 & 25.31 & 56.10 \\
\hline
\end{tabular}

There are significant differences in the number of citations received by papers depending on the type of collaborating country by $R \& D$ intensity level in all fields $(p<0.001)^{5}$ except in Mathematics. Articles co-authored with high R\&D countries receive a higher number of citations, while those developed in collaboration with low $R \& D$ countries tend to be less cited. The greatest influence of the type of collaborating country is found in the Social Sciences, Clinical Medicine and Multidisciplinary fields, where the articles co-authored with low R\&D countries received half the number of citations of those conducted in collaboration with high R\&D countries. The highest RCR corresponds in Mathematics to papers co-authored with countries whose R\&D intensity is similar to that of Spain but, as mentioned above, the differences were not statistically significant (figure 3a).

\footnotetext{
${ }^{5}$ Kruskal-Wallis test for non-parametric distributions
} 
Concerning publication journals, significant differences in NJP by type of collaborating country are observed across all fields $(p<0.001)$ and papers co-authored with high R\&D countries tend to be published in more prestigious journals (higher NJP), even in Mathematics. NJP relative to the country average by field is shown in figure $3 \mathrm{~b}$.

Figure 3. Relative impact of articles according to the level of R\&D investment of the collaborating country
a. Citations
b. Normalised Journal Position
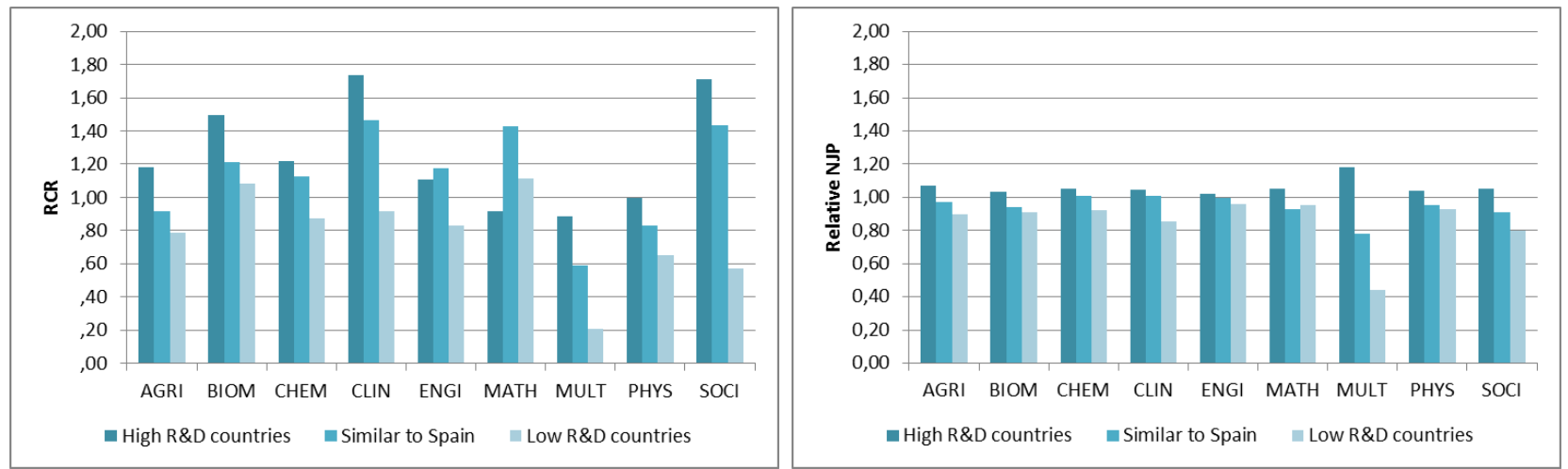

\subsection{Ordinal logistic regression analysis}

The relative citation rate variable is categorised in four classes as explained in the Methodology section. Ordinal regression analysis was performed to study the association between the relative citation rate and the following variables: type of country, country of the corresponding author, number of authors, number of references, normalised journal position and research field. The null hypothesis that there was no difference between the model without independent variables (only-intercept model) and the model with independent variables is rejected attending to the Omnibus test (Chisquare $=2,154.65 ; p<0.001$ ).

The ordinal logistic regression analysis output is presented in table 3 , where we can see the logistic coefficient $(B)$ for each predictor variable. The logistic coefficient is the expected amount of change in the logit for each one unit change in the predictor. The closer a logistic coefficient is to zero, the less influence the predictor has in predicting the logit. The table also displays the standard error, the Wald statistic and associated $\mathrm{p}$-values. The Wald statistic tests the null hypothesis that a particular parameter estimates is zero.

The 'exp' function is applied to the B coefficients to obtain "Exp(B)" (last column in table 3 ) which is the odds ratio associated with each predictor. We expect predictors which increase the logit to display $\operatorname{Exp}(B)$ greater than 1.0, those predictors which do not have an effect on the logit will display an $\operatorname{Exp}(B)$ of 1.0 and predictors which decrease the logit will have $\operatorname{Exp}(B)$ values less than 1.0.

The variables number of authors, number of references and NJP are significant and show an $\operatorname{Exp}(B)$ greater than one, which means that as they increase so does the likelihood of being in a higher level of the relative citation rate. The highest influence is 
observed for NJP, which shows the highest $\operatorname{Exp}(B)$. Concerning categorical variables, the $\operatorname{Exp}(B)$ provide information on the relative odds for each category in relation to the reference category holding all else constant. The reference categories are "Spain" for the country of the corresponding author; and "low R\&D country" for "type of country". Accordingly, the probability of being in a higher level of relative citation rate is higher for high and medium than for low R\&D countries.

To explore potential interfield differences in the influence of the variables "type of country" and "country of the corresponding author", we search for interactions between these variables and the fields. Only those interactions that are significant, which corresponds to Mathematics and Social Sciences, are maintained in the final model. Firstly, an interaction between "country of the corresponding author" and Mathematics emerges, so that in this field publications with a non-Spanish corresponding author are more likely to enjoy high relative citation rates $(\operatorname{Exp}(B)=1.388)$.

Moreover, an interaction between type of country and Social Sciences, on the one hand and type of country and Mathematics, on the other, can also be observed in table 3. For Social Sciences, the influence of the type of country resembles that observed in the general model without interactions, but here the positive influence of the high and medium $R \& D$ countries is stronger, as shown by the higher $\operatorname{Exp}(B)$ values. In the case of Mathematics, the highest positive influence on the relative citation rate is observed in the collaboration with medium $R \& D$ countries $(\operatorname{Exp}(B)=1.486)$. The negative influence of the collaboration with high $R \& D$ countries $(\operatorname{Exp}(B)=0.696)$ as compared with low $R \& D$ ones is a surprising result which needs to be interpreted with caution, taking into account that it can be a correction of the model with interactions with respect to the general model without them. A careful interpretation seems to be appropriate, but even in that case we can conclude that collaboration with high $R \& D$ countries is clearly less advantageous in Mathematics than in the rest of the fields. 
Table 3. Ordinal logistic regression model for relative citation rate

\begin{tabular}{|c|c|c|c|c|c|}
\hline Parameter & B & Std. Error & $\begin{array}{c}\text { Wald } \\
\text { Chi-square }\end{array}$ & Sig. & $\operatorname{Exp}(B)$ \\
\hline Thresholds & .569 & .0986 & 33.275 & .000 & 1.766 \\
\hline [crel=2.00] & 3.721 & .1073 & $1,202.594$ & .000 & 41.321 \\
\hline$[\mathrm{crel}=3.00]$ & 5.113 & .1126 & $2,062.054$ & .000 & 166.203 \\
\hline [Corresp.author=0] & .042 & .0428 & .962 & .327 & 1.043 \\
\hline [Corresp.author=1] & $0^{\mathrm{a}}$ & & & & 1 \\
\hline [Type of country=1] & .223 & .0537 & 17.274 & .000 & 1.250 \\
\hline [Type of country=2] & .187 & .0726 & 6.651 & .010 & 1.206 \\
\hline [Type of country=3] & $0^{\mathrm{a}}$ & & & & 1 \\
\hline Number of authors & .067 & .0118 & 32.785 & .000 & 1.070 \\
\hline Normalised Journal Position & 3.125 & .0963 & $1,054.147$ & .000 & 22.761 \\
\hline Number of references & .015 & .0008 & 331.149 & .000 & 1.016 \\
\hline AGRI_dummy & -.170 & .0571 & 8.897 & .003 & .843 \\
\hline BIOM_dummy & .148 & .0643 & 5.304 & .021 & 1.160 \\
\hline SOCl_dummy & -.511 & .2061 & 6.148 & .013 & .600 \\
\hline PHYS_dummy & -.377 & .0585 & 41.541 & .000 & .686 \\
\hline ENGI_dummy & -.065 & .0538 & 1.462 & .227 & .937 \\
\hline MATH_dummy & .009 & .1526 & .004 & .951 & 1.009 \\
\hline CLIN_dummy & .379 & .0809 & 21.996 & .000 & 1.461 \\
\hline MULT_dummy & -.662 & .2307 & 8.231 & .004 & .516 \\
\hline CHEM_dummy & -.239 & .0595 & 16.083 & .000 & .788 \\
\hline [Corresp.author $=0$ ] * MATH & .328 & .1410 & 5.408 & .020 & 1.388 \\
\hline [Corresp.author $=1$ ] * MATH & $0^{\mathrm{a}}$ & & & & 1 \\
\hline$[$ Type of country $=1]{ }^{*}$ MATH & -.363 & .1652 & 4.828 & .028 & .696 \\
\hline$[$ Type of country $=2]{ }^{*}$ MATH & .396 & .2182 & 3.299 & .069 & 1.486 \\
\hline [Type of country=3] * MATH & $0^{\mathrm{a}}$ & & & & 1 \\
\hline$[\text { Type of country }=1]^{*} \mathrm{SOCl}$ & 1.256 & .2227 & 31.824 & .000 & 3.512 \\
\hline [Type of country=2] ${ }^{*} \mathrm{SOCl}$ & .772 & .3167 & 5.946 & .015 & 2.165 \\
\hline [Type of country=3] * SOCl & $0^{\mathrm{a}}$ & & & & 1 \\
\hline
\end{tabular}

Corresponding author: $1=$ Spain; $0=$ Other country

Type of country: $1=$ high R\&D country; $2=$ similar to Spain; $3=$ low R\&D country.

a Reference category 


\section{Discussion}

\subsection{Impact gain from international collaboration}

Our results show differences by field in the collaboration pattern which are consistent with those previously described in the literature (Luukkonen, Persson and Sivertsen, 1992). The scientific output of Spain during our reference period produces a higher impact when it is internationally co-authored, with Clinical Medicine, Multidisciplinary, Biomedicine and Social Sciences as the fields which show a higher increase in their average citation scores when foreign authors participate in the research. The important role of coordinated multi-country research projects in Biomedicine and multicentre clinical trials in Clinical Medicine can be brought forward to explain the high impact of internationally co-authored papers in these fields (García-Romero et al., 2009). Concerning Multidisciplinary sciences, international collaboration tends to boost the already high impact of papers published in high prestigious journals such as Science or Nature (Jiménez-Contreras et al., 2010). Interestingly, the citation gain obtained from international collaboration in Physics is not very large in spite of the important role played by central research facilities in the field (e.g., CERN) and the high visibility usually described for this "mega-science" research. This result is consistent with the findings of Guerrero-Bote et al. (2013) concerning impact gain in internationally coauthored world papers in the field and can be partly explained by the high share of internationally co-authored papers in Physics, which tends to raise the average field impact and reduces the values of citation rates relative to this average.

\subsection{High and low $R \& D$ partners}

With a focus on bilateral collaboration, we observe that Spanish teams collaborate mainly with high R\&D countries in all fields. Scientifically advanced countries are more attractive partners since their research teams usually have more material and economic resources and enjoy greater scientific recognition, which is supposed to yield higher benefits to their partners. This is supported by the study of Gazni, Sugimoto and Didegah (2012) where world's countries were categorized in four different groups in terms of rate of scientific development. Additionally, an economic approach was also used by these authors, who observed that high-income countries were the core group in international collaboration and that they tend to be the main partner of other types of countries. Interestingly, in our study the highest percentage of papers in collaboration with low R\&D countries is found in Agriculture and Chemistry (29\% and 33\% respectively), maybe because Spain is a particularly "attractive" partner for low R\&D countries in these fields in which it shows a high specialisation or revealed comparative advantage (Harzing and Giroud, 2014).

\subsection{Factors influencing research impact}

On the basis of our regression analysis, the citations received by papers tend to increase with the number of authors, the number of references and the prestige of the publication journal. Larger teams have been previously associated with a higher research impact due to the collaboration of scientists with different specialisation profiles which may result in more original and consistent research (Bordons, Aparicio and Costas, 2013; Gazni and Didegah, 2011). Papers with a higher number of references are probably more comprehensive papers (McVeigh and Mann, 2009) and they are more likely to produce some impact on the community (Haslam et al., 2008). Moreover, a higher number of references per paper has been described for "top" scientists in a previous study (Costas, Van Leeuween and Bordons, 2012). Finally, a positive correlation between the prestige of journals and citations received by papers has been reported in the literature (see for example, Didegah and Thelwall, 2013). 
Our study also shows that the number of citations received by papers tends to grow with the R\&D intensity of collaborating countries, with this trend being particularly conspicuous in Social Sciences. The more positive influence of collaboration with high R\&D countries has been previously described for a specific biomedical discipline in Spain (Bordons, Aparicio and Costas, 2013) and for different areas in Latin American countries (De Filippo, Aparicio and Gómez, 2009). Moreover, greater gains were described from collaboration with high-impact countries in a study focused on world publications during 2003-2009 (Guerrero-Bote, Olmeda-Gómez and Moya-Anegón, 2012).

Why do social sciences articles benefit so much from collaboration with high R\&D countries? The fact that very low citation scores are observed in this field for papers performed by a single institution ("no collaboration"), maybe due to the more local orientation of some of the topics of research, can be an explanatory factor since it makes the boost associated to international co-authorship more evident.

The case of Mathematics is intriguing, since joint research with high R\&D countries seems to be less rewarding than in other areas in terms of citations. The fact that research in Mathematics does not rely on expensive infrastructures so much as in other fields, leads us to think that it could be easier for intermediate or low R\&D countries to attain excellence in this field. Moreover, it could also be the case that low or intermediate R\&D countries become high R\&D investing countries in this specific field. In our study, Spain obtains its highest impact in Mathematics when collaborating with intermediate $R \& D$ countries. The examination of this set of papers allows us to identify Italy and China as the most beneficial partners (RCR $>1$ in both cases) and it is interesting to note that both countries show high specialisation in Mathematics, that is, their share of publications in the field is higher than in all science fields (Harzing and Giroud, 2014). Furthermore, among the low R\&D countries that more often collaborate with Spain, Latin America is prominent as a regional partner (Brazil, Argentina and Mexico) probably for language-sharing reasons, along with a number of Eastern European Countries, such as Poland, Hungary, Romania or Ukraine, which have been described as specialised in Mathematics (Glanzel, 2001; Harzing and Giroud, 2014). Among low R\&D countries the collaboration with Romania and Ukraine shows a particularly high impact, which again points at the positive effect of specialised partners.

Finally, our results show that whether or not Spain is the country of the corresponding author, it does not have a significant influence on the impact of research in most fields. This contrasts with previous findings on this issue which have suggested a higher impact for papers lead by scientifically advanced countries - mostly measured through first authorship- (Jiménez-Contreras et al., 2010; Van Leeuwen, 2009). However, it should be kept in mind that our results concern papers in international bilateral collaboration while the abovementioned studies dealt with all type of internationally coauthored papers, including bilateral and multilateral links. As the number of partners in a research venture increases so does the probability of uneven participation and the importance of the leader in steering and coordinating the investigation. This could help to explain the higher influence of the identity of the leader country in the final impact of research developed under an international multilateral collaboration scheme.

However, the positive influence of having a corresponding country other than Spain is observed in Mathematics regardless of the type of country involved in the research. Do Spanish teams tend to collaborate in particular with strong teams from other countries? According to the ISI Essential Indicators of Thomson Reuters, a high specialisation and a high impact relative to world average is described for Spain in Mathematics (20032013) and this can improve Spain's options when selecting partners from abroad. 


\subsection{Policy implications}

International collaboration is a strategic factor which may contribute to optimise the impact of Spanish publications in most fields. Although the highest citation gain is obtained from multilateral collaboration, bilateral collaboration is also associated with an increase in impact, and it accounts for a large share of internationally co-authored papers, amounting up to $80 \%$ in some fields, such as Mathematics. Multilateral international collaboration is more often the result of "top-down" initiatives such as those funded under the European Commission Framework Programmes (Mattsson et al., 2010). Bilateral collaboration is more likely to be funded by national sources on the basis of bilateral agreements and promoted by scientists themselves (Gorraiz, Reimann and Gumpemberger, 2012). Moreover, contacts established through bilateral collaboration schemes can serve as a stepping stone for wider future collaborations. In summary, each type of collaboration has its own role to play in the advancement of science and deserves specific studies.

Our results show that in most fields the highest citation gain is obtained from the collaboration with high $R \& D$ countries. However, it should be noted that the collaboration with low R\&D countries is also relevant. As described by Wagner et al. (2001) in a study based on interviews with principal researchers in collaborative projects, the expertise of individual scientists and shared scientific interests are the most important reasons for collaborating, irrespective of the scientists' country of origin. Scientists from low R\&D countries can become strategic partners due to their unique geographical, ecological or social conditions; but also because they may be strong in specific disciplines or specialities, in particular in areas of observational and theoretical science that require less costly equipment (e.g. mathematics). As mentioned by Wagner et al. (2001), institutions and individuals with world-class expertise can be found in otherwise not very scientifically advanced countries.

However, a gain in impact is not the only purpose of international collaboration (Adams, Gurney and Marshall, 2007). Scientifically advanced countries also collaborate with less advanced countries to address specific problems in what has been called "research aid for development" or "capacity building" (Wagner et al., 2001), which aims at contributing to increase scientific capacity in the more peripheral countries. International collaboration fosters the access of less advanced countries to funding, facilities and networks and enables them to participate in world science. On the one hand, international collaboration may have a positive effect on the national science system of these countries as well as on their economic growth and social welfare. On the other hand, science is increasingly global and the participation of all countries is needed to address global challenges more effectively (The Royal Society, 2011).

Finally, we would like to mention that international scientific mobility may play an important role in the development of international collaboration. On the one hand, mobile researchers may continue working and co-authoring with researchers in their home institution; on the other hand, returned scientists may maintain collaborative links with their former host organisation (Jonkers and Cruz-Castro, 2013). It is difficult to identify mobile scientists in publications, but around $13 \%$ of papers in our study included authors with two different addresses, one in Spain and a second one in another country, which probably correspond to mobile scientists who include both the temporary and the permanent address in their publications. There is one caveat concerning this double assignment since it entails the possibility of detecting "artificial international collaboration", but our data suggest that in more than $50 \%$ of the cases 
there was a real international collaboration in the relevant papers ${ }^{6}$ and that Spanish scientists abroad do probably facilitate the expansion of the collaborative network of their home institution, although further research would be required in order to establish this point. The extent to which highly competent and skilled mobile Spanish scientists located in foreign top-institutions might contribute to the higher impact observed for internationally co-authored papers also remains an intriguing topic for further scrutiny. Research results on these issues may inform and support public policies concerning international mobility, which is being encouraged to foster the access of scientists to knowledge, skills and networks (Edler et al., 2011).

\subsection{Future research}

Our paper provides insights for future research. Firstly, the influence of the thematic specialisation of countries is mentioned in our study, but an in-depth analysis of the issue by countries and fields would be required. Secondly, the role of the international mobility of scientists in the establishment of international collaboration between different types of countries as well as in the impact of the resulting publications also will benefit from further research. Finally, extending this study to multilateral collaboration would be of interest, since the latter has shown the highest increase in the last decade (Bordons, Aparicio and Costas, 2013; Mattsson et al., 2010); it is a major factor to be reckoned with for the achievement of the highest impact in research; and can be a useful instrument for capacity building in less scientifically advanced countries.

\section{Acknowledgements.}

Financial support from the Spanish National R\&D Plan (Research project CSO2008-06310) is acknowledged. We are very grateful to Isabel Gómez, for her comments on a previous version of this paper and to Laura Barrios and José Manuel Rojo for their statistical advice. The comments of two anonymous referees which contribute significantly to improve the content of the paper are also acknowledged.

\section{References}

Adams, J., Gurney, K. A., and Marshall S. (2007). Patterns of international collaboration for the UK and leading partners. A report commissioned by the UK Office of Science and Innovation. Leeds: Evidence Ltd.

Bordons, M.; Aparicio, J. and Costas, R. (2013). Heterogeneity of collaboration and its relationship with research impact in a biomedical field. Scientometrics, 96(2), 443-466.

Bordons, M.and Barrigón, S. (1992). Bibliometric analysis of publications of Spanish pharmacologists in the SCI (198489). II. Contribution to subfields other than "Pharmacology \& Pharmacy" (ISI). Scientometrics, 25(3), 425-446.

Costas, R.; van Leeuwen, T.N. and Bordons, M. (2012). Referencing patterns of individual researchers: do top scientists rely on more extensive information sources? Journal of the American Society for Information Science and Technology, 63(12), 2433-2450.

De Filippo, D.; Aparicio, J. and Gómez, I. (2009). Measuring the benefits of International collaboration. A case study of the relationship between Latin-American and European countries. B.Larsen and J.Leta, Eds. Proceedings of the 12th International Conference of the ISSI (pp.920-921), Brasil: BIREMEPPAHO/WHO and Federal University of Rio de Janeiro.

Didegah, F. and Thelwall, M. (2013). Which factors help authors produce the highest impact research? Collaboration, journal and document properties. Journal of Informetrics, 7, 861-873.

\footnotetext{
${ }^{6}$ Since we observed that: a) apart from the author with two addresses (one in Spain and a second one in other country) there was at least one co-author with a Spanish affiliation and one co-author with a foreign affiliation in a given paper; or b) Spanish and foreign authors' names coexisted in a given paper.
} 
Edler, J.; Fier, H.; Grimpe, C. (2011). International scientist mobility and the locus of knowledge and technology transfer. Research Policy, 40(6), 791-805.

Eurostat (2014), Statistics explained. Retrieved July 15, 2014 from http://epp.eurostat.ec.europa.eu/statistics explained/index.php/Glossary:R \%26 D intensity

Frandsen, T.F.and Nicolaisen, J. (2010). What is in a name? Credit assignment practices in different disciplines. Journal of Informetrics, 4, 608-617.

García-Romero, A.; Navarrete-Cortés, J.; Escudero, C.; Fernández-López, J.A. and Chaichío-Moreno, JA. (2009). Measuring the influence of clinical trials citations on several bibliometric indicators. Scientometrics, 80(3), 749-762.

Garson, G.D. (2013). Logistic regression: binary and multinomial. Asheboro, USA: Statistical Associates Publishing.

Gazni, A.and Didegah, F. (2011). Investigating different types of research collaboration and citation impact: a case study of Harvard University's publications. Scientometrics, 87(2), 251-265.

Gazni, A.; Sugimoto, C.R.and Didegah, F. (2012). Mapping world scientific collaboration: authors, institutions and countries. Journal of the American Society for Information Science and Technology, 63(2), 323-335.

Glänzel, W. (2001). National characteristics in international scientific co-authorship relations. Scientometrics, 51(1), 69115.

Glanzel, W.; Schubert, A. (2004). Analysing scientific networks through co-authorship. In: H.F. Moed et al. (eds). Handbook of Quantitative Science and Technology Research, 257-276. Dordrecht: Kluwer Academic Publishers.

Goldfinch, S.; Dale, T. and DeRouen, K (2003). Science from the periphery: publication, collaboration and "periphery effects" in article citation rates of the New Zealand Crown Research Institutes 1995-2000. Scientometrics, 57(3), 321337.

Gómez, I.; Bordons, M.; Morillo, F.; Moreno, L.; Aparicio, J. and González-Albo, B. (2012). La actividad científica del CSIC a través del Web of Science. Estudio bibliométrico del período 2007-2011 (pp.1-1400), Madrid: IEDCYT, CCHS, CSIC. http://hdl.handle.net/10261/65287.

González-Albo, B.; Aparicio, J.; Moreno, L.; Bordons; M. (2013). Leaders and partners in international collaboration and their influence on research impact. En: Gorraiz, J.; Schielbel, E.; Gumpenberger, C.; Hörlesberger, M.; Moed, H. Proceedings of the 14th International Conference of the ISSI (pp.2044-2047), Viena.

Gorraiz, J.; Reimann, R. and Gumpenberger, C. (2012). The importance of bilateral and multilateral differentiation in the assessment of international collaboration -a case study for Austria and six countries. Scientometrics, 91(2), 417-433.

Guerrero-Bote, V.P.; Olmeda-Gómez, C. and Moya-Anegón, F. (2013). Quantifying the benefits of international scientific collaboration. Journal of the American Society for Information Science and Technology, 64(2), 392-404.

Haslam, N., Ban, L., Kaufmann, L., Loughnan, S., Peters, K., Whelan, J. and Wilson, S. (2008). What makes an article influential? Predicting impact in social and personality psychology. Scientometrics, 76(1), 169-185.

Harzing, A.W. and Giroud, A. (2014). The competitive advantage of nations: an application to academia. Journal of Informetrics, 8, 29-42.

Jiménez-Contreras, E.; Torres-Salinas, D.; Ruíz-Pérez, R. and Delgado-López-Cozar, E. (2010). Investigación de excelencia en España: ¿protagonistas o papeles secundarios? Medicina Clínica (Barc), 134(2), 76-81.

Jonkers, K.; Cruz-Castro. L. (2013). Research upon return: The effect of international mobility on scientific ties, production and impact. Research Policy, 42, 1366-1377.

Katz, S. and Hicks, D. (1997). How much is a collaboration worth? A calibrated bibliometric model. Scientometrics, 40(3), 541-554.

Katz, S. and Martin, B. (1997). What is research collaboration? Research Policy, 26(1), 1-18.

Luukkonen, T., Persson, O., and Sivertsen, G. (1992). Understanding patterns of international scientific collaboration. Science, Technology and Human Values, 17, 101-126.

Marusic, A.; Bosnjak, L. and Jeroncic, A (2011) A systematic review of research on the meaning, ethics and practices of authorship across scholarly disciplines. PLOS ONE, 6(9), e23477. doi:10.1371/journal.pone.002347.

Mattsson, P., Sundberg, C. J., and Laget, P. (2011). Is correspondence reflected in the author position? A bibliometric study of the relation between corresponding author and byline position. Scientometrics, 87(1), 99-105.

Mattsson, P.; Laget, P.; Vindefjard, A.N. and Sundberg, C.J.(2010). What do European research collaboration networks in life sciences look like? Research Evaluation, 19(5), 373-384. 
McVeigh, M.E. and Mann, S.J. (2009). The Journal Impact Factor Denominator: Defining Citable (Counted) Items. The Journal of the American Medical Association, 302(10), 1107-1109.

Narin, F.; Stevens, K. and Whitlow, E. (1991). Scientific cooperation in Europe and the citations of multinationally authored papers. Scientometrics, 21(3), 313-323.

Nomaler, O.; Frenken, K. and Heimeriks, G. (2013). Do more distant collaborations have more citation impact? Journal of Informetrics, 7, 966-971.

Reagans, R. and Zuckerman, E.W. (2001). Diversity and productivity: the social capital of corporate R\&D teams. Organization Science, 12(4), 502-517.

The Royal Society (2011). Knowledge, networks and nations: global scientific collaboration in the 21st century. London: Royal Society.

Van Leeuwen, T.N. (2009). Strength and weakness of national science systems. A bibliometric analysis through cooperation patterns. Scientometrics, 79(2), 389-408.

Van Raan, A. (1997). Science as an international enterprise. Science and Public Policy, 24(5), 290-300.

Waltman, L. (2012). An empirical analysis of the use of alphabetical authorship in scientific publishing. Journal of Informetrics, 6, 700-711.

Wagner, C.S.; Brahmakulam, I.; Jackson, B.; Wong, A. and Yoda, T. (2001). Science and technology collaboration: Building capacities in developing countries. Santa Monica, CA: RAND.

Wren, J.D.; Zozak, K.Z.; Johnson, K.R.; Deakyne, S.J.; Schilling, L.M. and Delavalle, R.P. (2007). The write position. A survey of perceived contributions to papers based on byline position and number of authors. EMBO Reports, 8(11), 988991. 